\title{
The sandwich technique for repair of pectus carinatum and excavatum/carinatum complex
}

\author{
Hyung Joo Park, Kyung Soo Kim \\ Department of Thoracic and Cardiovascular Surgery, Seoul St. Mary's Hospital, College of Medicine, The Catholic University of Korea, 222 \\ Banpo-Daero, Seocho-Gu, Seoul, South Korea \\ Correspondence to: Hyung Joo Park, MD, PhD, FCCP, Professor and Chief. Department of Thoracic and Cardiovascular Surgery, Seoul St. Mary's Hospital, \\ College of Medicine, The Catholic University of Korea, 222 Banpo-Daero, Seocho-Gu, Seoul 06591, South Korea. Email: hyjpark@catholic.ac.kr.
}

Background: Simple external compression of pectus carinatum seems to have its limitations, particularly the repair of asymmetric pectus carinatum or excavatum/carinatum complex. We devised the sandwich technique (press-molding) to remodel the entire chest wall. The purpose of this study is to introduce the sandwich technique and appraise the early results.

Methods: Between January 2007 and January 2016, 523 consecutive patients with pectus carinatum and its variants were analyzed retrospectively. No patients were excluded during the study period. The sandwich 1 and 2 techniques using the internal and external pectus bars were for pectus carinatum repair. Modified techniques using the external string and the internal bar were to treat the lower costal flare (the flare-buster) and focal protuberances (the magic string) in pectus excavatum repair. Statistical analyses were carried out using paired and unpaired $t$-test or Wilcoxon signed rank tests.

Results: The sandwich repair with the external and internal bars was applied to 58 pectus carinatum patients: seven symmetric (12.1\%), 14 asymmetric (24.1\%), and 37 carinatum-excavatum complex (63.8\%). After pectus excavatum repair, 426 patients had the flare-buster and 39 patients received the magic string. The sandwich 1 technique achieved near-complete resolution of carinatum in 52 patients (86.2\%). The sandwich 2 technique accomplished almost symmetric configuration with no residual carinatum in all six cases.

Conclusions: The sandwich technique using the external and internal bars seems to be effective in treating asymmetric pectus carinatum and complex excavatum/carinatum deformities. The flare-buster and the magic string effectively relieve the costal flare and focal protuberances in pectus excavatum repair.

Keywords: Pectus carinatum; pectus excavatum; minimally invasive repair; press-molding; sandwich technique

Submitted Mar 07, 2016. Accepted for publication Aug 08, 2016.

doi: 10.21037/acs.2016.08.04

View this article at: http://dx.doi.org/10.21037/acs.2016.08.04

\section{Introduction}

Minimally invasive surgical repair of the pectus carinatum has not been widely accepted. Compression of the chest wall protrusion by using a pectus bar was introduced by Abramson (1), but simple external compression was not successful in asymmetric pectus carinatum or excavatum/ carinatum complex. Therefore, we devised the concept of remodeling the entire anterior chest wall for cases of carinatum or combined type chest wall deformities through a press-molding technique, using external and internal bar sandwiching. The purpose of this study is to introduce the technical details of our approach and evaluate the early results of repair of pectus carinatum and its variants.

\section{Methods}

\section{Patient population}

Out of the cohort of a single surgeon's (HP) experience 
A

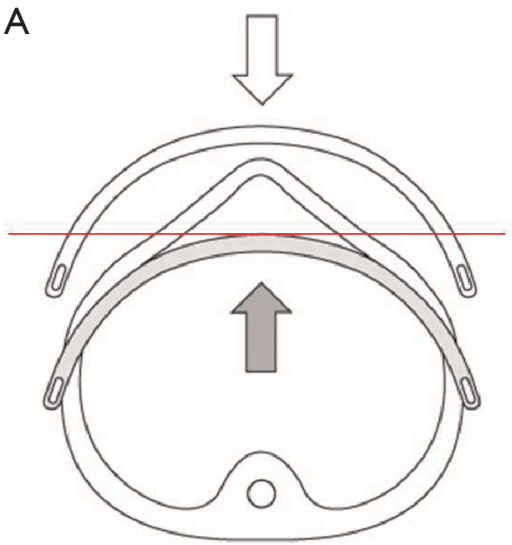

B

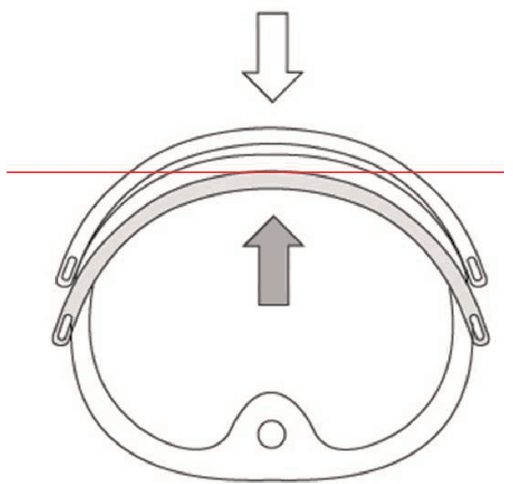

Figure 1 Repair of symmetric pectus carinatum: press-molding the anterior chest wall by sandwiching between the external bar (white) and the internal bar (gray). (A) pre-repair; (B) post-repair.

with 2,640 pectus repair patients, there were 58 patients who received the sandwich technique for repair of pectus carinatum and its variants since 2007, 426 patients who had the flare-buster since 2011, and 39 patients who underwent the magic string procedure since 2015. The sandwich technique was applied to 16 patients with pectus carinatum, and 42 with pectus carinatum/excavatum complex. The mean age of the patients was 12.26 years (range, 5 to 23 years). Forty-eight patients (82\%) were male.

We included the data for these 523 consecutive patients from January 2007 to January 2016 in this retrospective analysis. Patients with all types of morphologies and age groups were included, with no patient in our cohort excluded from the analysis.

To verify the efficacy of the flare-buster, 40 patients, who were operated on in 2010, before the year we developed the flare-buster, were enrolled as a control group, and the flare angles were compared with the flare-buster group.

\section{The principle of the technique}

Simple external compression of the chest wall to correct pectus carinatum aims to reduce the chest wall protrusion. However, the chest wall will not reform evenly, because there will be undesirable compression of the chest wall next to the carinated portion. Thus, simple external compression should not be used for asymmetric deformities or combined excavatum/carinatum complexes.

We hypothesize that the chest wall needs to be pressmolded for proper pectus carinatum repair; that is, the chest wall should be supported from internally, whilst externally compressing the carinatum to evenly remodel the chest wall.

\section{The indication for the sandwich technique}

We do not have a numerical guideline for the indication of surgery so far. Our primary approach for simple pectus carinatum has been brace therapy. Once a patient has a significant pectus carinatum, we decide whether surgical repair is appropriate based on a consultation with the patient and family at each occasion. Surgical repair is indicated in cases of symmetric carinatum that are not suitable for bracing; asymmetrical chest wall deformities; and combined excavatum and carinatum deformities.

We find that the sandwich technique is indicated for the following chest wall deformities: pectus carinatum, symmetric (Figure 1) or asymmetric (Figure 2); pectus excavatum/carinatum complex (Figure 3); pectus excavatum with focal protrusions; and lower costal flare relief. The flare angle is defined as the angle between the sternal line and flare line (Figure 4).

This technique was originally applied to pediatric patients but continues to extend to all age groups.

\section{Surgical techniques of the procedure}

We utilize two bars: the internal and external bars, to achieve the necessary internal support to oppose the external compression of the chest wall (press-molding, the sandwich technique).

To begin with, we insert the internal bar first, followed by external bar compression (the sandwich 1 technique). 


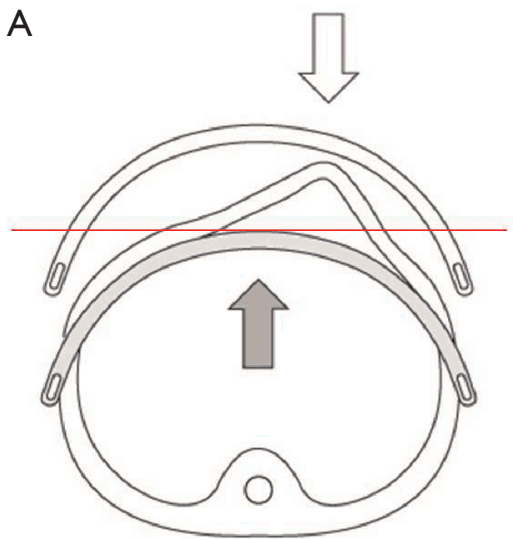

B

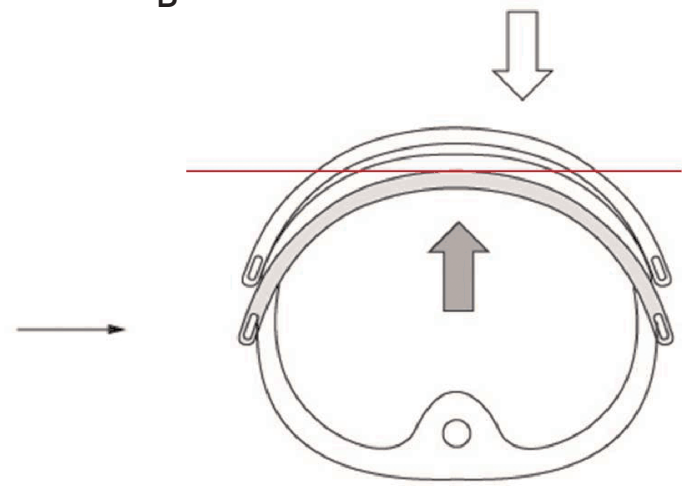

Figure 2 Repair of asymmetric (eccentric) pectus carinatum: press-molding the anterior chest wall by sandwiching between the external bar (white) and the internal bar (gray). (A) pre-repair; (B) post-repair.

A

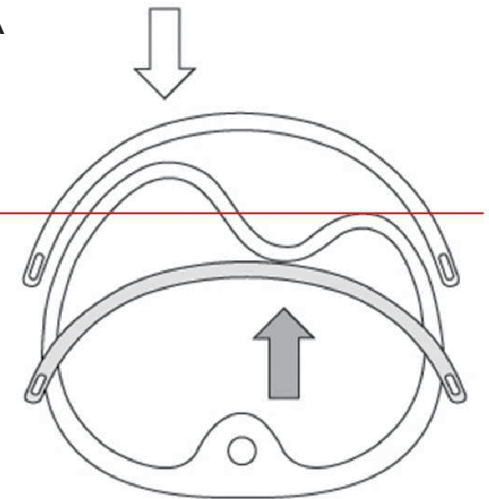

B

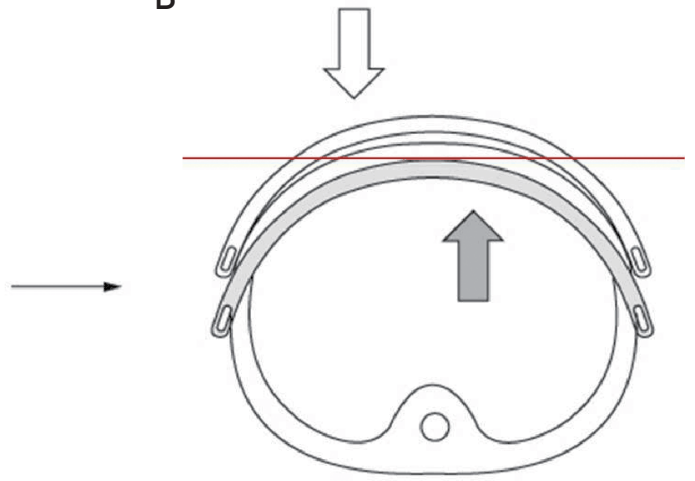

Figure 3 Repair of pectus carinatum/excavatum complex: press-molding the anterior chest wall by sandwiching between the external bar (white) and the internal bar (gray). (A) pre-repair; (B) post-repair.
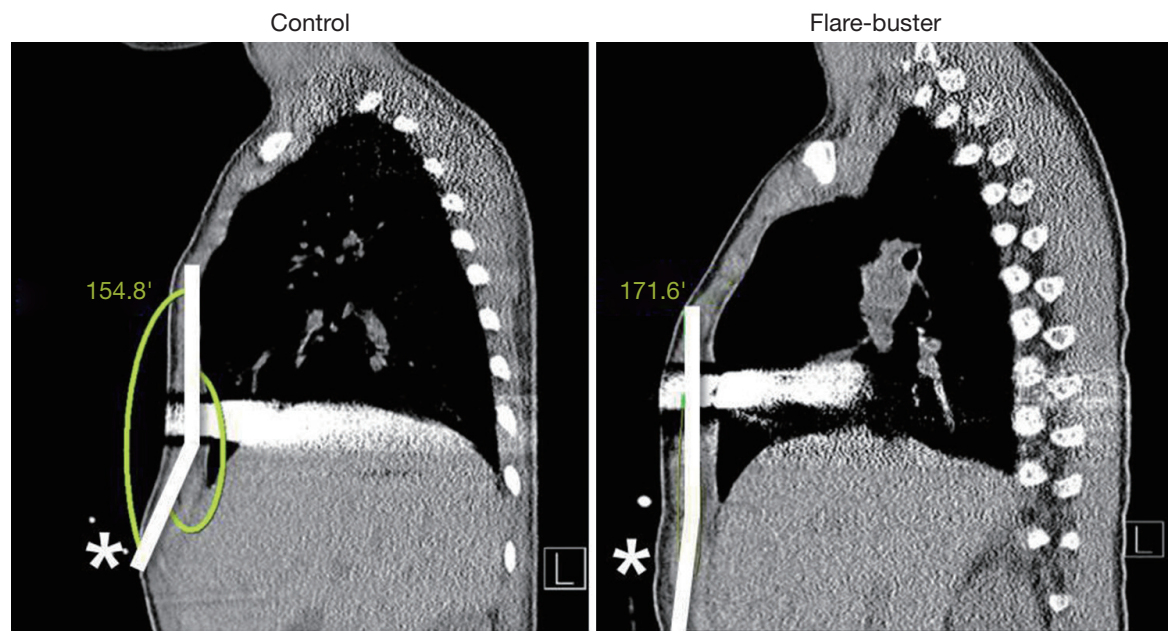

Figure 4 Comparison of the flare angles between the control group (without flare-buster) and the flare-buster group. The flare angle is defined as the angle between sternal line and flare line (the white bold lines). *, lower costal flare. 
Later, however, we found that performing the external bar compression first is more efficient in compressing the carinatum deformity. Accordingly, we adopted the sandwich 2 technique in 2015: compressing the carinatum first, followed by lifting the depressions.

Our modification of the sandwich technique, utilizing a string instead of the external pectus bar, inspired the development of the flare-buster in 2013 and the magic string in 2015.

\section{The sandwich 2 technique (Video 1)}

First, the targets are marked on the chest wall. The primary target, for the external bar, is on the top of the carinatum, the point of compression. The secondary target, for the internal bar, is the depressed portion of the chest wall, often located on both lower costal areas. Commonly, the secondary targets are identified better when manually pressing down on the carinatum portion.

The internal bar is shaped to fit the chest wall configuration; the convexity of the bar accounts for the points of the depression. The pectoscope is introduced through a small chest incision to create the path for the internal bar. We leave the guide (24 Fr. chest tube) for the internal bar in place without inserting the bar. The external bar bears a flat segment where it meets the carinated part, to provide more compression. The subcutaneous tunnel is created to pass through the apex of the chest wall protrusion for the external compression on the carinatum. To fix the external bar to the corresponding ribs, we place the pericostal wire sutures on each side of the chest, utilizing the "through-the-skin suture technique (2)." The pericostal wires are tied to the end holes of the bar while compressing the carinatum using the table mounted compressor (Omni Crane System, Primemed, Seoul, South Korea) (3).

The internal bar is passed via the previously placed guide and elevates the depressed part of the chest wall by rotating it. Finally, the external and internal bars are connected to the bridge plate (4).

\section{The Flare-Buster (Video 2)}

The remaining lower costal flare after pectus excavatum is compressed with strings: No.2 PDS for children or No.5 Ethibond for older patients. After completing pectus excavatum repair, the top of the costal flare on both sides are marked. The subcutaneous tunnel is created across the highest points of costal flares from one side to the other.
Then the string is passed and tied to the end holes of the bar while the flare is manually compressed.

\section{The magic string (Video 3)}

After completion of pectus excavatum repair, focal protrusions are identified. With the same technique as the flare-buster, the string is passed over the top of these protrusions via the subcutaneous dissection and compressed by tying the string to the bar.

\section{Outcome analysis}

To evaluate outcomes of repair, the changes in the computerized tomography (CT) index: Haller Index and Asymmetry Index were analyzed for the sandwich technique. The efficacy of the flare-buster technique was examined by comparison of pre and post-repair flare angles.

\section{Statistical analysis}

Statistical analyses were carried out with the SPSS software package (version 10.0, SPSS, Chicago, IL, USA). Continuous variables were compared using unpaired, and paired t-test or Wilcoxon signed rank test as appropriate. $\mathrm{P}$ values $<0.05$ were considered to be significant.

\section{Results}

The sandwich repair (1 or 2) using press-mold with the external and internal bars was applied to 58 patients. The morphological features of the patients were five $(8.6 \%)$ symmetric pectus carinatum, $11(18.9 \%)$ asymmetric pectus carinatum, and $42(72.4 \%)$ pectus carinatum/excavatum complex cases.

The length of follow-up of the patients was: 3 months to 9 years (median, 48 months) for the sandwich technique; 3 to 52 months (median, 37 months) for the flare-buster; and 3 to 9 months (median, 5 months) for the magic string technique.

The result of repair was near complete resolution of carinatum in 50 patients $(96.2 \%)$, but in two patients, the carinatum was not effectively compressed by the sandwich 1 technique due to its rigidity. The sandwich 2 technique achieved an almost symmetric configuration with no residual carinatum in all six cases.

In pectus carinatum patients who underwent a sandwich 2 repair, the Haller index increased from $2.04 \pm 0.34$ to $2.54 \pm 0.29$ $(\mathrm{P}<0.001)$. The asymmetry index decreased from $1.05 \pm 0.10$ 

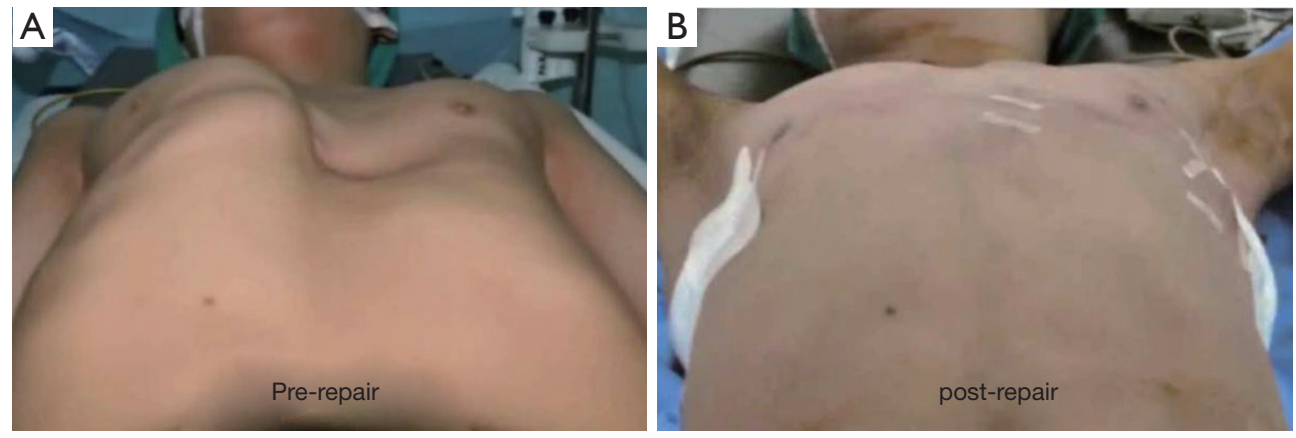

Figure 5 Pre and post-repair pictures of a 17-year-old boy with an extreme pectus excavatum/carinatum complex. The repair was carried out using the sandwich technique.

to $1.01 \pm 0.01(\mathrm{P}=0.005)$ after repair. In pectus excavatum/ carinatum complex patients, Haller index reduced from $5.29 \pm 1.75$ to $2.74 \pm 0.42(\mathrm{P}<0.001)$. The asymmetry index decreased from $1.09 \pm 0.08$ to $1.02 \pm 0.01(\mathrm{P}=0.012)$ after repair.

No serious complications related to the procedure were observed, except for one pleural effusion and one wound hematoma. Thirty-three patients $(56.9 \%)$ had pectus bar removal, and their results were favorable.

The flare-buster was applied to 426 patients of pectus excavatum. The mean age of the patients was 7.8 years (range, 3 to 50 years). Seventy six percent of the patients was male. The morphological features of the chest wall deformity were: symmetric in $307(72.1 \%)$ patients, eccentric in 92 (21.6\%), and unbalanced in 24 (5.6\%).

The efficacy of the flare-buster was verified using measurements of flare angles before and after repair (Figure 4). There was a significant difference in flare angles between the control and the flare-buster group: $163.04 \pm 4.58$ degrees in the control group versus $174.97 \pm 2.31$ degrees in the flare-buster group $(\mathrm{P}<0.001)$.

The magic string was applied to 39 patients with pectus excavatum. The mean age was 9.03 years (range, 3 to 26 years). Thirty patients $(76.9 \%)$ were male. The morphological features of the chest wall deformities were: symmetric in $24(66.7 \%)$ patients, eccentric in four (10.3\%), and unbalanced in eight (20.5\%). The magic string relieved focal protrusions and was assessed to be effective according to the operator's judgment.

There were no complications related to the flare-buster or the magic string procedure.

\section{Discussions}

We judge the methods that simply pressing down on the carinatum with a pectus bar $(1,5)$ or a brace (6-9) would not be ideal for pectus carinatum correction, even in simple symmetric types. Our reasoning for this belief was our observation that when we compressed a portion of the chest wall, the surrounding areas are also inevitably compressed. To avoid this and improve the repair, we believe that the forces need to be exerted on both sides of the chest wall. Therefore, instead of a simple compression of any methods, we invented the technique of the "press-mold" by sandwiching two bars inside and outside of the chest wall.

Moreover in our observations, the pure symmetric pectus carinatum was rare. Instead, mostly the chest wall protrusions were located eccentrically or combined some form of focal chest wall depressions $(8,9)$. Therefore, we have not tried to distinguish precisely which is carinatum or which is excavatum. Our approach involves recognizing pectus deformities as a whole, and trying to convert them to a normal configuration $(10,11)$. When the deformity is a combined excavatum/carinatum, we apply sandwich techniques (press molding) using a bar or string.

We first started our press-molding approach in 2007 for cases of severe chest wall deformities involving a carinatumexcavatum complex. After obtaining an excellent result in a case of extreme pectus excavatum/carinatum complex (Figure 5), we were convinced that the sandwich approach is the ideal method of correcting all types of chest wall protrusions. We therefore applied this technique to a total of 58 patients including those with pectus carinatum and mixed deformities of excavatum and carinatum.

This approach was extended to relieve the lower costal flare, the flare-buster, in 2013. The lower costal flare invariably accompanied with the pectus excavatum due to the unique feature of the pectus excavatum deformity: the scaphoid costal cartilage configuration. It has no 
physiological significance but often causes cosmetic dissatisfaction. The simple maneuver adapted from the sandwich technique using heavy strings created an effective relief of the flares. For this purpose, instead of the external pectus bar, heavy strings are used to compress the costal protrusions.

We frequently encounter accompanied focal protuberances in cases of the eccentric (Grand Canyon type) or unbalanced pectus excavatum. These hidden knobs at the brim of the chest wall excavation become apparent once the excavated wall is lifted. To resolve this issue, we applied the string sandwich technique, the magic string, to eliminate any residual focal chest wall protuberances that emerge after pectus excavatum repair. The procedure is identical to the flare-buster. We found that this strategy contributed significantly to an improved cosmetic outcome.

The sandwich technique, flare-buster, and the magic string were initially thought to be possible only in pediatric patients, but their use continues to extend to all age groups. Young adult patients have a resistant costal cage, but with the aid of the Omni crane compression system, the carinatum can be molded to a greater degree (3).

\section{Limitations of the study}

The limitations of this study include its short follow-up and small sample size, particularly for the sandwich technique. We will have to see the late results of the patients after bar removal.

\section{Conclusions}

The sandwich technique enabled us to correct pectus carinatum deformities, including asymmetric and pectus excavatum/carinatum complexes, by remodeling the entire anterior chest wall. The flare-buster and magic string techniques further enhanced the repair by eliminating any residual protrusions or depressions of the chest wall. Our techniques seem promising in resolving pectus carinatum as well as undesirable lower costal flare and focal protrusions. However, the value of this press-molding approach warrants further evaluation of longer-term outcomes.

\section{Acknowledgements}

None.

\section{Footnote}

Conflicts of Interest: The authors have no conflicts of interest to declare.

\section{References}

1. Abramson H, D'Agostino J, Wuscovi S. A 5-year experience with a minimally invasive technique for pectus carinatum repair. J Pediatr Surg 2009;44:118-23; discussion 123-4.

2. Park HJ, Jeong JY, Kim KT, et al. Hinge reinforcement plate for adult pectus excavatum repair: a novel tool for the prevention of intercostal muscle strip. Interact Cardiovasc Thorac Surg 2011;12:687-91.

3. Park HJ, Kim KS, Lee S, et al. A next-generation pectus excavatum repair technique: new devices make a difference. Ann Thorac Surg 2015;99:455-61.

4. Park HJ, Kim KS, Moon YK, et al. The bridge technique for pectus bar fixation: a method to make the bar unrotatable. J Pediatr Surg 2015;50:1320-2.

5. Yüksel M, Bostanci K, Evman S. Minimally invasive repair of pectus carinatum using a newly designed bar and stabilizer: a single-institution experience. Eur J Cardiothorac Surg 2011;40:339-42.

6. Haje SA. Pectus carinatum successfully treated with bracing--a case report. Int Orthop 1995;19:332-3.

7. Martinez-Ferro M, Fraire C, Bernard S. Dynamic compression system for the correction of pectus carinatum. Semin Pediatr Surg 2008;17:194-200.

8. Kang DY, Jung J, Chung S, et al. Factors affecting patient compliance with compressive brace therapy for pectus carinatum. Interact Cardiovasc Thorac Surg 2014;19:900-3.

9. Cohee AS, Lin JR, Frantz FW, et al. Staged management of pectus carinatum. J Pediatr Surg 2013;48:315-20.

10. Park HJ, Lee SY, Lee CS, et al. The Nuss procedure for pectus excavatum: evolution of techniques and early results on 322 patients. Ann Thorac Surg 2004;77:289-95.

11. Park HJ, Jeong JY, Jo WM, et al. Minimally invasive repair of pectus excavatum: a novel morphology-tailored, patient-specific approach. J Thorac Cardiovasc Surg 2010;139:379-86.

Cite this article as: Park HJ, Kim KS. The sandwich technique for repair of pectus carinatum and excavatum/carinatum complex. Ann Cardiothorac Surg 2016;5(5):434-439. doi: 10.21037/acs.2016.08.04 